\title{
Baseline MDCT findings after prosthetic heart valve implantation provide important complementary information to echocardiography for follow-up purposes
}

\author{
Dominika Suchá $^{1}$ • Steven A. J. Chamuleau ${ }^{2}$ - Petr Symersky ${ }^{3}$ - Matthijs F. L. Meijs ${ }^{4}$. \\ Renee B. A. van den Brink ${ }^{5}$ • Bas A. J. M. de Mol ${ }^{6}$ - Willem P. Th. M. Mali ${ }^{1}$. \\ Jesse Habets $^{1}$ • Lex A. van Herwerden ${ }^{7}$ • Ricardo P. J. Budde R,8 $^{1,8}$
}

Received: 13 April 2015 /Revised: 27 May 2015 / Accepted: 17 June 2015 /Published online: 16 October 2015

(C) The Author(s) 2015. This article is published with open access at Springerlink.com

\begin{abstract}
Objectives Recent studies have proposed additional multidetector-row CT (MDCT) for prosthetic heart valve (PHV) dysfunction. References to discriminate physiological from pathological conditions early after implantation are lacking. We present baseline MDCT findings of PHVs 6 weeks post implantation.
\end{abstract}

A comment to this article is available at http://dx.doi.org/10.1007/ s00330-015-3937-3.

Electronic supplementary material The online version of this article (doi:10.1007/s00330-015-3918-6) contains supplementary material, which is available to authorized users.

Dominika Suchá

d.sucha@umcutrecht.nl

1 Department of Radiology, University Medical Center Utrecht, Huispostnummer E01.132, Heidelberglaan 100, PO Box 85500, 3508 GA Utrecht, The Netherlands

2 Department of Cardiology, University Medical Center Utrecht, Utrecht, The Netherlands

3 Department of Cardiothoracic Surgery, VU Medical Center, Amsterdam, The Netherlands

4 Department of Cardiology, Thoraxcentrum Twente, Medisch Spectrum Twente, Enschede, The Netherlands

5 Department of Cardiology, Academic Medical Center, Amsterdam, The Netherlands

6 Department of Cardiothoracic Surgery, Academic Medical Center, Amsterdam, The Netherlands

7 Department of Cardiothoracic Surgery, University Medical Center Utrecht, Utrecht, The Netherlands

8 Department of Radiology, Erasmus University Medical Center, Rotterdam, The Netherlands
Methods Patients were prospectively enrolled and TTE was performed according to clinical guidelines. 256-MDCT images were systematically assessed for leaflet excursions, image quality, valve-related artefacts, and pathological and additional findings. Results Forty-six patients were included comprising 33 mechanical and 16 biological PHVs. Overall, MDCT image quality was good and relevant regions remained reliably assessable despite mild-moderate PHV-artefacts. MDCT detected three unexpected valve-related pathology cases: (1) prominent subprosthetic tissue, (2) pseudoaneurysm and (3) extensive pseudoaneurysms and valve dehiscence. The latter patient required valve surgery to be redone. TTE only showed trace periprosthetic regurgitation, and no abnormalities in the other cases. Additional findings were: tilted aortic PHV position $(n=3)$, pericardial haematoma $(n=3)$ and pericardial effusion $(n=3)$. Periaortic induration was present in 33/40 (83\%) aortic valve patients.

Conclusions MDCT allowed evaluation of relevant PHV regions in all valves, revealed baseline postsurgical findings and, despite normal TTE findings, detected three cases of unexpected, clinically relevant pathology.

Key Points

- Postoperative MDCT presents baseline morphology relevant for prosthetic valve follow-up.

- $83 \%$ of patients show periaortic induration 6 weeks after aortic valve replacement.

- MDCT detected three cases of clinically relevant pathology not found with TTE.

- Valve dehiscence detection by MDCT required redo valve surgery in one patient.

- MDCT is a suitable and complementary imaging tool for follow-up purposes.

Keywords Multidetector-row computed tomography $\cdot$ Heart valve prosthesis · Echocardiography · Diagnostic imaging · Reference standard 


$\begin{array}{ll}\text { Abbreviations } \\ \text { BMI } & \text { Body mass index } \\ \text { BPM } & \text { Beats per minute } \\ \text { EOA } & \text { Effective orifice area } \\ \text { LVOT } & \text { Left ventricular outflow tract } \\ \text { MDCT } & \text { Multidetector-row computed tomography } \\ \text { PHV } & \text { Prosthetic heart valve } \\ \text { RCA } & \text { Right coronary artery } \\ \text { TTE } & \text { Transthoracic echocardiography } \\ \text { VTI } & \text { Velocity time integral }\end{array}$

\section{Introduction}

Current cardiology guidelines recommend transthoracic echocardiography (TTE) 6-12 weeks after valve replacement surgery as a baseline for adequate patient follow-up $[1,2]$. For mechanical valves, TTE is only repeated in case of suspected dysfunction, whereas for biological valves annual TTE assessment should be planned after the first 5 years [1]. As additional modalities for the evaluation of prosthetic valve dysfunction, transoesophageal echocardiography and cinefluoroscopy are advised. However, these imaging modalities often cannot identify the cause of obstruction or the extent of endocarditisrelated pathology [3, 4]. Several preliminary studies have shown the incremental value of ECG-gated multidetectorrow computed tomography (MDCT) for the evaluation of patients with prosthetic heart valves (PHV) [4-8]. Recently, MDCT was added to the American College of Cardiology and European Society of Cardiology guidelines as a potential additional imaging modality for patients with suspected PHV thrombus [1, 2], pannus [1] or endocarditis [2]. Before MDCT can be fully implemented in daily clinical practice, it is essential to be able to discriminate normal from pathological conditions. As proposed with TTE imaging, follow-up results should be compared to the acquired baseline references [1]. Currently, normal references for MDCT findings early after PHV implantation are lacking. The purpose of this study was to evaluate the baseline MDCT imaging characteristics 6 weeks after PHV implantation and report incidental pathological findings.

\section{Methods}

\section{Study sample}

A prospective study was conducted to acquire standardized TTE and MDCT images 6 weeks after PHV implantation. Patients were consecutively enrolled between June 2010 and September 2013 after surgical aortic or mitral heart valve replacement in the University Medical Center Utrecht and Academic Medical Center Amsterdam, The Netherlands. The inclusion criterion was successful implantation of one of the common surgical PHV types of the participating institutions: St. Jude Medical, Sorin Carbomedics, ON-X, Sorin Bicarbon, Carpentier-Edwards Perimount or Sorin Mitroflow. Exclusion criteria consisted of (1) known allergy to contrast media, (2) other contraindications for contrast use such as an estimated glomerular filtration rate $<60 \mathrm{ml} / \mathrm{min} / 1.73 \mathrm{~m}^{2}$, (3) contraindication for MDCT imaging, (4) possible pregnancy and (5) refusal to be informed about additional MDCT findings.

This study was approved by the Institutional Review Board and all patients gave written informed consent before inclusion.

\section{Transthoracic echocardiography (TTE) imaging and analysis}

TTE was performed on the principal investigation site (Utrecht) 6 weeks after implantation according to routine follow-up guidelines and by using standard clinical echocardiographic equipment. Images were evaluated by one independent observer (MM; cardiologist) with 5 years' experience in cardiac imaging. A second observer (SC; imaging cardiologist) with 8 years' experience in PHV imaging evaluated all cases of abnormalities and pathological findings for a consensus score. Both observers were blinded for MDCT results. PHV assessment was conducted according to the guidelines $[1,9]$ and included measurements of the mean and peak transprosthetic gradients, velocity time integrals (VTI), calculation of effective orifice area (EOA) and evaluation of regurgitation. The VTI was measured through the left ventricular outflow tract (LVOT) and across the PHV (aortic or mitral). The EOA was calculated using the LVOT diameter and Doppler continuity equation for all PHVs $[10,11]$, as the pressure half-time method is not preferred in mitral PHVs [9]. Available guidelines specified per PHV manufacturer and PHV size were used as reference [9]. Periprosthetic regurgitation was scored on a visual scale as none, trace, mild, moderate or severe. Prosthetic regurgitation was scored as normal for PHV, trace, mild, moderate or severe. Additional (pathological) findings were reported. Left ventricular function was measured on available 3D full volume dataset, or $2 \mathrm{D}$ images by the Simpson biplane method and scored as normal (>55\%), mild dysfunction (45-55\%), moderate dysfunction $(30-45 \%)$ or severe dysfunction $(<30 \%)$. If measurements were not possible, the left ventricular function was estimated.

\section{MDCT imaging}

MDCT images were acquired on the same day as TTE imaging using a 256-slice MDCT system (iCT, Philips Healthcare, Best, The Netherlands). The acquisition protocol consisted of a non-enhanced prospectively ECG-triggered acquisition of the PHV region with the following parameters: collimation $128 \times 0.625 \mathrm{~mm}$, gantry rotation time $270 \mathrm{~ms}$, tube voltage 
$120 \mathrm{kV}$ and tube current-time product $30 \mathrm{mAs}$. For contrastenhanced imaging a triphasic injection protocol was applied using iodinated contrast agent (Ultravist $300 \mathrm{mg} / \mathrm{ml}$; Bayer Healthcare Pharmaceutical Inc.). First, the contrast agent was administered followed by a mixture of contrast agent and saline in 30/70 ratio, respectively and a saline flush at last (mean flow rate 6-6.7 cc/s). Flow and volume injection were weight dependent and a threshold of 100 Hounsfield units in the descending aorta was used for acquisition. No beta blockers were administered for heart rate control. The contrast-enhanced retrospectively ECG-gated acquisition of the cardiac region was performed according to the following protocol: collimation $128 \times 0.625 \mathrm{~mm}$, gantry rotation time 270 or $330 \mathrm{~ms}$, pitch 0.16 or 0.18 , tube voltage $120 \mathrm{kV}$ and tube current-time product 600 or $700 \mathrm{mAs}$. The rotation time and pitch were dependent on the heart rate $(<62,62-72$ or $>72$ beats per minute; bpm) and the tube current-time product on the patients' body weight ( $65-80$ or $>80 \mathrm{~kg}$ ). Images were reconstructed at each $10 \%$ phase of the RR-interval with a 0.45 increment and $0.9 \mathrm{~mm}$ slice thickness. The dose-length product $\left(\mathrm{mGy}^{*} \mathrm{~cm}\right)$ extracted from the MDCT dose reports and the conversion factor for adult chest MDCT examinations $\left(0.0145 \mathrm{mSv} / \mathrm{mGy}^{*} \mathrm{~cm}\right)$ were multiplied to acquire the effective per patient radiation dose (mSv) [12].

\section{Multidetector-row computed tomography (MDCT) analysis}

Images were analysed on a dedicated workstation (Extended Brilliance Workstation v4.5, Philips Healthcare, Best, The Netherlands). For each PHV, the best systolic and diastolic imaging phase was selected. Both phases were used to assess the image quality, PHV-related artefacts and leaflet motion. Image quality and PHV-related artefacts were assessed for five PHVrelated regions as published previously [13, 14]: the supraprosthetic, periprosthetic, subprosthetic, prosthetic and valve leaflet region. Image quality was scored on a 4-point scale as follows: (1) non-diagnostic with no discernible detail widely beyond the prosthesis, (2) moderate with no details $<5 \mathrm{~mm}$ of the prosthesis, (3) good with some structure details $<5 \mathrm{~mm}$ of the prosthesis and (4) excellent visualization with perfect structure details. PHV-related artefacts were scored as: (1) virtually none, (2) mild artefacts $<5 \mathrm{~mm}$ beyond the prosthesis, (3) moderate artefacts $>5 \mathrm{~mm}$ beyond the prosthesis and (4) severe artefacts beyond the border of the valve chamber. For mechanical PHVs, the leaflet opening and closing angles were measured [15] and cine images reconstructed for evaluation of symmetrical leaflet excursions. Pericardial effusion was evaluated and scored as physiological, mild or moderate. The fat tissue surrounding the aorta and aortic PHVs was assessed and tissue induration was reported when present. Polytetrafluoroethylene felt pledgets implanted during valve surgery were reported when visible. All PHVs were systematically evaluated and scored for unexpected pathology such as thrombus, pannus, vegetations, mycotic aneurysms, biological leaflet thickening, pseudoaneurysms, PHV dehiscence and PHV angulation in relation to the LVOT axis. For all patients possible additional findings were reported as well. One observer (DS) with 2 years' experience in PHV imaging performed all MDCT measurements independently. Image quality, artefacts and pathology were assessed by a second observer (RB) with 7 years' experience additionally for a consensus score. Both observers were blinded for TTE results.

\section{Statistical analysis}

For analysis of the acquired data descriptive statistics were applied using SPSS version 20.0 for Windows. As measures of central tendency and dispersion, the mean \pm standard deviation (SD) or median [interquartile range; IQR] are provided, based on the data distribution. For summary statistics the number and percentage are provided.

\section{Results}

A total of 68 patients were consecutively included after valve surgery. Of these, 16 patients decided upon study withdrawal at 6 weeks, one patient died, one patient showed an impaired renal function, one patient underwent MDCT for clinical reasons and three patients were excluded because of imaging protocol violation. Hence, 46 patients with 49 valves were included. Patient characteristics are presented in Table 1. Median time between valve replacement surgery and TTE/ MDCT imaging was 44 days [IQR 41-52]. TTE and MDCT examinations were performed on the same day in 45 patients and with an interval of 2 days in one patient.

\section{TTE}

The mean heart rate during TTE examinations was $74 \pm$ $16 \mathrm{bpm}$. Six of 46 patients had atrial fibrillation during examination. Left ventricular ejection fraction was normal in 30 patients, showed mild dysfunction in seven patients, moderate dysfunction in six patients and severe dysfunction in three patients. Mean EOA was $2.00 \pm 0.64$ for aortic and $2.05 \pm$ 0.41 for mitral PHVs. An overview of all TTE results on PHV assessment is presented in Table 2. One patient with a Carbomedics 27-mm PHV showed mild to moderate prosthetic regurgitation but this was considered clinically insignificant. Hence, TTE results on prosthetic and periprosthetic regurgitation did not show any pathological findings requiring short-term interventions. Pericardial effusion was not present or scored as physiological $(<2 \mathrm{~mm})$ in 42 patients on TTE imaging at 6 weeks. One patient showed pericardial effusion of $7 \mathrm{~mm}$ and three patients had greater pericardial effusion 
Table 1 Patient characteristics at baseline

\begin{tabular}{|c|c|c|}
\hline Study subjects & $\begin{array}{l}\text { Patients } \\
\mathrm{N}=46\end{array}$ & \\
\hline Age, mean \pm SD & $61 \pm 12$ & \\
\hline Male, $n(\%)$ & $37(80 \%)$ & \\
\hline Prior myocardial infarction & $1(2 \%)$ & \\
\hline Prior cardiac surgery & $8(17 \%)$ & \\
\hline $\mathrm{BMI}$, mean $\pm \mathrm{SD}$ & $27.3 \pm 4.8$ & \\
\hline BSA & $1.98 \pm 0.22$ & \\
\hline Concomitant bypass surgery & $10(22 \%)$ & \\
\hline Concomitant other procedure & $15(33 \%)$ & \\
\hline \multicolumn{3}{|l|}{ Medication at discharge } \\
\hline Vitamin $\mathrm{K}$ antagonists & $43(94 \%)$ & \\
\hline Antiplatelet drugs & $16(35 \%)$ & \\
\hline Rate control drugs & $39(85 \%)$ & \\
\hline PHV type implanted, n (\%) & Aortic, $\mathrm{N}=40(82 \%)$ & Mitral, $N=9(18 \%)$ \\
\hline St. Jude Medical & $8(20 \%)$ & $4(44 \%)$ \\
\hline Carbomedics & $11(28 \%)$ & $1(11 \%)$ \\
\hline $\mathrm{ON}-\mathrm{X}$ & $3(8 \%)$ & $2(22 \%)$ \\
\hline Sorin Bicarbon & $3(8 \%)$ & $1(11 \%)$ \\
\hline Perimount (biological) & $11(28 \%)$ & $1(11 \%)$ \\
\hline Mitroflow (biological) & $4(10 \%)$ & - \\
\hline \multicolumn{3}{|l|}{ PHV size } \\
\hline $21 \mathrm{~mm}$ & $2(5 \%)$ & - \\
\hline $23 \mathrm{~mm}$ & $18(45 \%)$ & - \\
\hline $25 \mathrm{~mm}$ & $13(33 \%)$ & $2(22 \%)$ \\
\hline $27 \mathrm{~mm}$ & $6(15 \%)$ & $2(22 \%)$ \\
\hline $29 \mathrm{~mm}$ & $1(3 \%)$ & $1(11 \%)$ \\
\hline $31 \mathrm{~mm}$ & - & $3(33 \%)$ \\
\hline $33 \mathrm{~mm}$ & - & $1(11 \%)$ \\
\hline
\end{tabular}

$B M I$ body mass index, $B S A$ body surface area, $P H V$ prosthetic heart valve, $S D$ standard deviation

$(12,12$ and $13 \mathrm{~mm})$. An example of good quality TTE images for a biological and mechanical PHV is presented in Fig. 1.

\section{MDCT}

The mean effective radiation dose was $11.3 \pm 1.1 \mathrm{mSv}$. Heart rate during MDCT examinations was known in 38/46 patients and was $72 \pm 16 \mathrm{bpm}($ mean $\pm \mathrm{SD})$. Two patients showed arrhythmias during MDCT acquisition, 36 patients had a normal sinus rhythm. The optimal MDCT reconstructions were 30 $40 \%$ systolic and 75-80-90\% diastolic phases (Electronic Supplementary Material Table S1). Results on image quality and artefacts are presented in Electronic Supplementary Material Tables S2 and S3, classified by manufacturer and anatomical region. For aortic valves in general, moderate artefacts and image quality were found for the periprosthetic region, and good image quality with only mild artefacts for the other regions. For mitral valves, mild to moderate artefacts with moderate to good image quality were present for the periprosthetic, subprosthetic and prosthetic regions and good image quality with mild artefacts for the supraprosthetic region. All mechanical valves showed good to excellent leaflet image quality whereas the biological Mitroflow and Perimount showed moderate and non-diagnostic leaflet image quality, respectively (Fig. 1). Mechanical leaflet excursions are presented in Table 3. The differences in angles between both leaflets of one PHV were assessed and a maximal median difference of $4^{\circ}$ for opening and $7^{\circ}$ for closing angles was found. Leaflet motion as assessed on cine images showed symmetrical leaflet excursions in all $(n=33)$ mechanical PHVs.

\section{Additional findings}

MDCT detected moderate pericardial effusion in three patients, the same patients as those detected by TTE. MDCT also showed a slight aortic PHV angulation in three patients. Prominent haematoma at periaortic and/or pericardial position 
Table 2 Transthoracic echocardiography results: patient characteristics 6 weeks after prosthetic valve implantation

\begin{tabular}{lll}
\hline TTE & Aortic PHV=40 & Mitral PHV=9 \\
\hline Effective orifice area*, mean \pm SD & $2.00 \pm 0.64$ & $2.05 \pm 0.41$ \\
Mean transprosthetic gradient, median [IQR] & $9.4[6.0-12.7]$ & $5.8[4.1-6.6]$ \\
Peak transprosthetic gradient & $17.5[12.2-27.1]$ & $14.7[7.7-17.4]$ \\
Periprosthetic regurgitation, $n(\%)$ & & $9(100 \%)$ \\
None & $38(95 \%)$ & - \\
Trace & $1(3 \%)$ & - \\
Mild & $1(3 \%)$ & - \\
Moderate & - & - \\
Severe & - & - \\
Prosthetic regurgitation & & - \\
Normal for PHV & $38(100 \%)$ & - \\
Trace & $1(3 \%)$ & - \\
Mild & - & - \\
Moderate & $1(3 \%)$ & \\
Severe & - &
\end{tabular}

PHV prosthetic heart valve, TTE transthoracic echocardiography

* Based on the Doppler continuity equation and measured left ventricular outflow tract diameter

$\uparrow$ Graded according to clinical routine based on mean and peak gradients, effective orifice area, cardiac output and available PHV type and size specific reference values

was present in three patients (Fig. 2). Most importantly, the periaortic fat tissue in patients after aortic valve replacement showed induration in 33 patients. Of those, two patients showed severe periaortic fat tissue induration (Fig. 2f).
Fig. 1 Example of biological and mechanical prosthetic heart valve imaging. Transthoracic

echocardiography images $(\mathbf{a}, \mathbf{c})$ and multidetector-row computed tomography images $(\mathbf{b}, \mathbf{d})$ of a normal functioning Perimount 25-mm biological valve $(\mathbf{a}, \mathbf{b})$ and a normal functioning Carbomedics 23-mm mechanical heart valve (c, d) in the aortic position
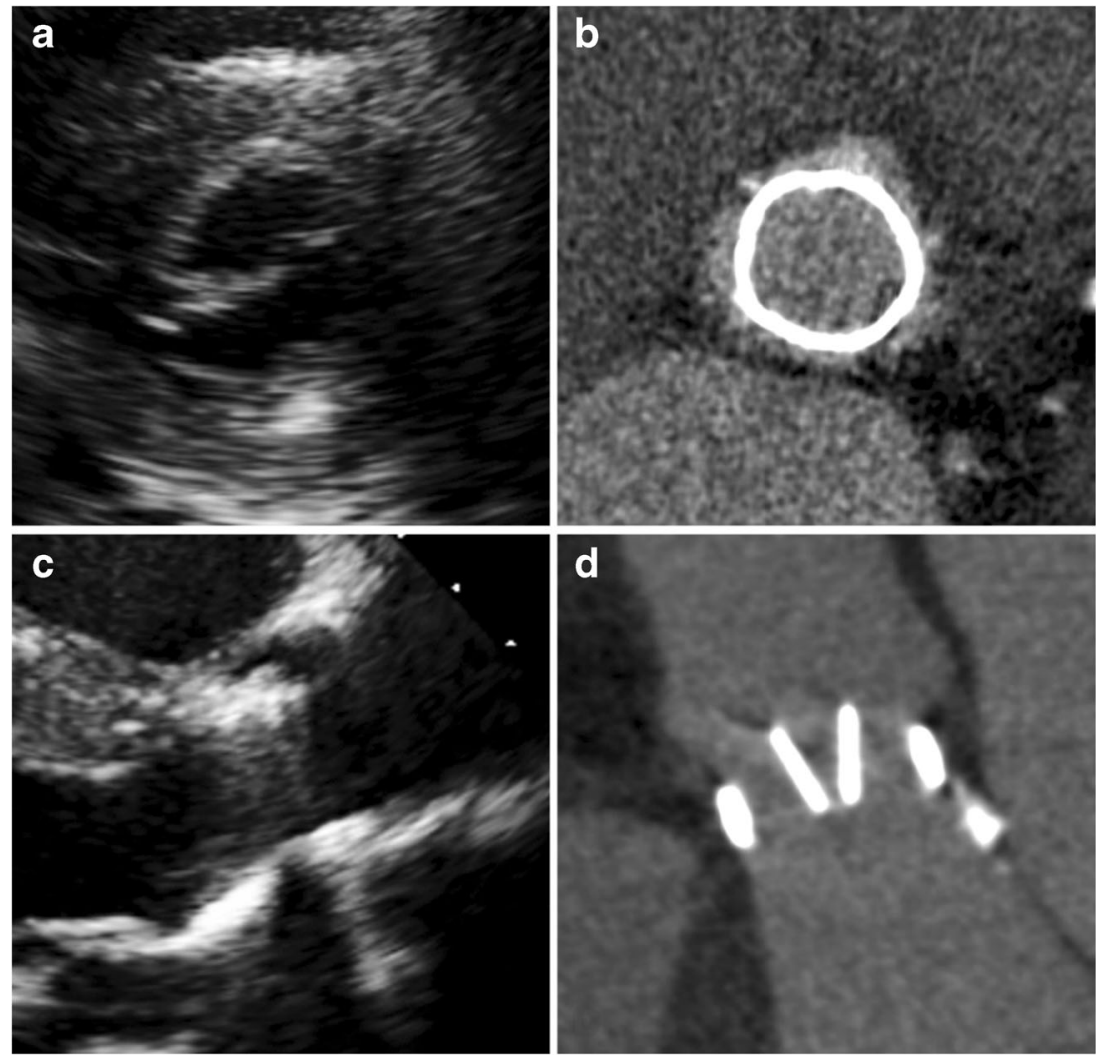
Table 3 Multidetector-row computed tomography mechanical valve leaflet angles as measured and compared to manufacturers' values

\begin{tabular}{|c|c|c|c|c|c|c|c|c|c|c|}
\hline & \multicolumn{5}{|c|}{ Opening angles $\left({ }^{\circ}\right)^{*}$} & \multicolumn{5}{|c|}{ Closing angles $\left({ }^{\circ}\right)^{*}$} \\
\hline & Aortic & $\Delta$ leaflets $\dagger$ & Mitral & $\Delta$ leaflets & MFR & Aortic & $\Delta$ leaflets $\dagger$ & Mitral & $\Delta$ leaflets & MFR \\
\hline St. Jude, $19-25 \mathrm{~mm}$ & $84(82-88)$ & $4(1-6)$ & $\mathrm{n} / \mathrm{a}$ & $\mathrm{n} / \mathrm{a}$ & 85 & $30(26-34)$ & $5(3-7)$ & $\mathrm{n} / \mathrm{a}$ & $\mathrm{n} / \mathrm{a}$ & 30 \\
\hline St. Jude, $27-31 \mathrm{~mm}$ & $85(83-87)$ & $2(1-4)$ & $83(80-85)$ & $3(1-5)$ & 85 & $23(22-27)$ & $3(0-5)$ & $23(22-27)$ & $2(0-5)$ & 25 \\
\hline Carbomedics & $80(72-82)$ & $2(0-3)$ & $73(72-74)$ & $2(-)$ & 78 & $24(20-28)$ & $3(0-7)$ & $24(22-27)$ & $5(-)$ & 25 \\
\hline Sorin Bicarbon & $79(78-80)$ & $1(1)$ & $78(77-78)$ & $1(-)$ & 80 & $21(19-22)$ & $1(1-3)$ & $20(19-20)$ & $1(-)$ & 20 \\
\hline On-X & $79(76-84)$ & $4(1-6)$ & $76(75-80)$ & $4(2-6)$ & 90 & $40(39-42)$ & $1(0-2)$ & $40(39-41)$ & $1(1)$ & 40 \\
\hline
\end{tabular}

MFR manufacturer, $n / a$ not available

* Median and total range (minimum-maximum) in degrees provided

$\dagger$ Median difference between the both leaflet angles of a specific prosthetic heart valve in each single patient

\section{Pathology}

MDCT found PHV-related pathology in three patients. First, MDCT detected prominent subprosthetic tissue in one patient with an aortic biological Perimount $23 \mathrm{~mm}$ (Fig. 3). Differential diagnosis was a retracted annulus, native valve remnant or small thrombus. Second, a pseudoaneurysm (approximately $22 \times 9 \times 13 \mathrm{~mm}$ ) was found on the supraprosthetic site near the former left and non-coronary cusp in a patient with an aortic biological Perimount $25 \mathrm{~mm}$, requiring follow-up
(Fig. 4). In addition, a malignant coronary anomaly was revealed in this patient showing the right coronary artery (RCA) deriving from the left ostium and coursing between the aorta and pulmonary trunk. Since MDCT showed RCA tapering, clinical evaluation and cardiac stress testing was required. Third, MDCT detected extended pseudonaneurysm formation and valve dehiscence in a patient with an aortic mechanical St. Jude $23 \mathrm{~mm}$ (Fig. 5). This patient eventually had to undergo redo surgery. In this patient, TTE only detected a trace of periprosthetic regurgitation stated as clinically not relevant.
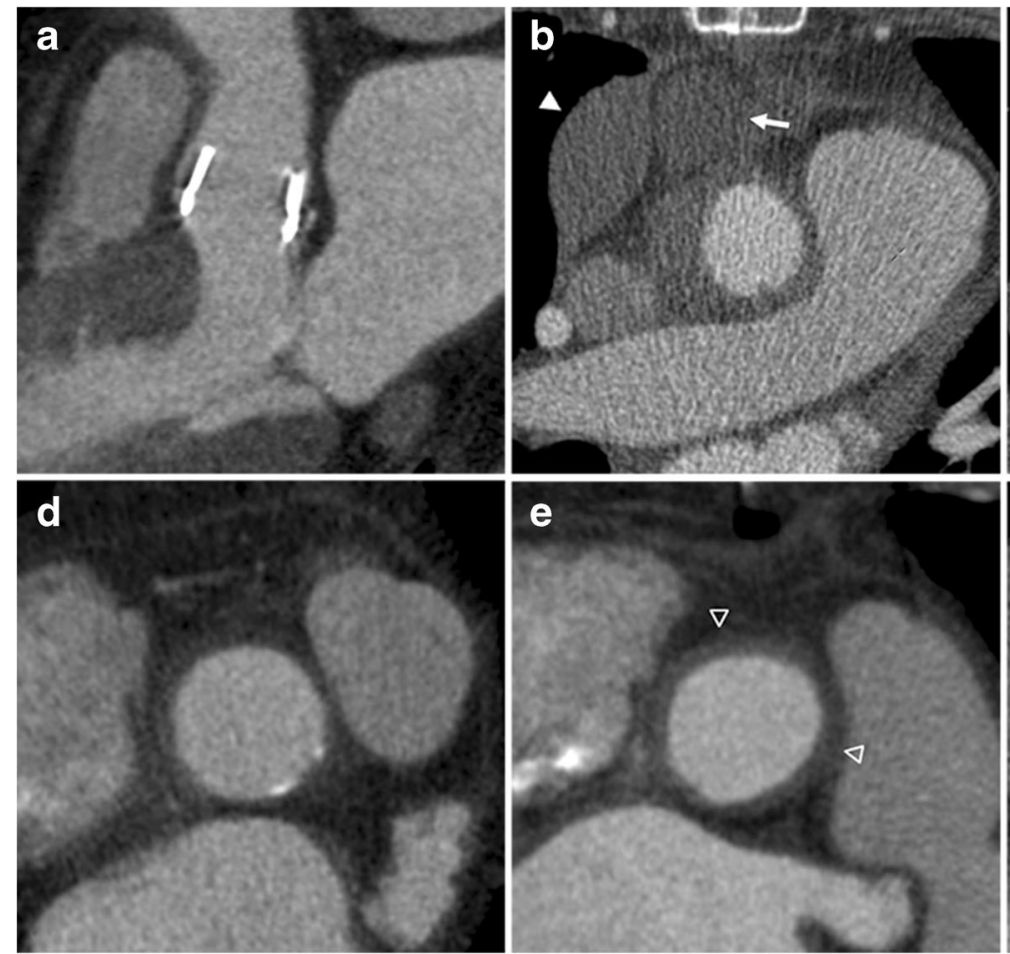
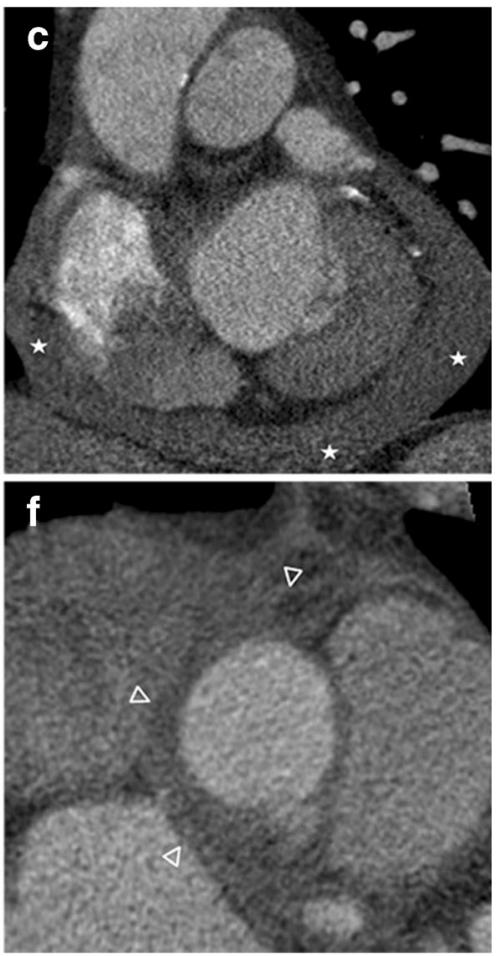

Fig. 2 Additional findings with multidetector-row computed tomography. (a) Example of a patient with a slightly tilted position of an aortic Perimount prosthesis. (b) Prominent pleural haematoma (35 Hounsfield Units; arrowhead) and pericardial haematoma (31 Hounsfield Units; arrow) in a patient 6 weeks after implantation of the aortic St. Jude valve. (c) Prominent pericardial effusion (stars) at 6 weeks' follow-up after biological Perimount implantation in the aortic position. (d-f) Multidector-row computed tomography (MDCT) images of the proximal ascending aorta in the short axis view in a patient without evident induration of the periaortic fat tissue (d), with moderate induration (e; empty arrowheads) and with severe induration of the periaortic tissue (f; empty arrowheads) 


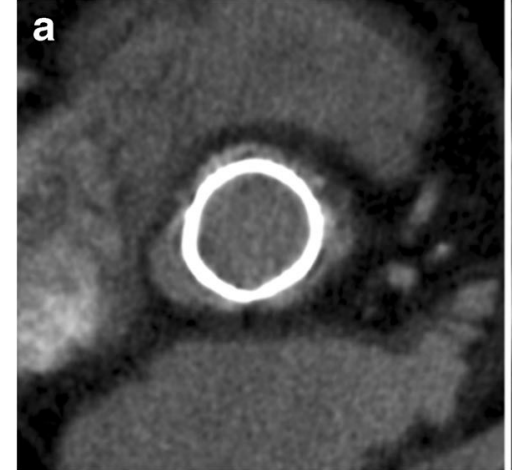

Fig. 3 Unexpected pathology detected with MDCT imaging. Multidector-row computed tomography (MDCT) images of a 69-yearold male patient 6 weeks after aortic Perimount 23-mm implantation (ac). Note the presence of the subprosthetic hypodense tissue (arrowhead) on both the in-plane (b) and the perpendicular plane image (c).

After the MDCT-based diagnosis, the patient underwent additional multimodality imaging [16]. Table 4 provides an overview of all PHV morphology as detected by MDCT.

\section{Discussion}

The current study demonstrates that MDCT is a feasible and valuable tool for PHV patient follow-up. We presented the baseline MDCT imaging characteristics and PHV morphology shortly after valve replacement surgery. The major finding was the detection of three cases of unexpected but clinically important valve-related pathology that were missed by routine TTE imaging. Furthermore, MDCT showed that in the early postoperative stage $83 \%$ of aortic valve patients present induration of the periaortic fat tissue, which can be considered a normal finding at this stage.

In this study, the presence of moderate valve-related artefacts did not hamper the image quality. Regions that are most relevant for clinical evaluation of PHV-related pathology such as the periprosthetic and subprosthetic region remained visible
Differential diagnosis: retracted annulus, native valve remnant or small thrombus. Transthoracic echocardiography did not detect any abnormalities in this patient. The stars in the image indicate the presence of polytetrafluoroethylene felt pledgets

and reliably assessable despite the presence of artefacts. In patients with a high body mass index (BMI), TTE imaging may be challenging and result in poor image quality whereas MDCT imaging may be hampered by higher image noise levels. In this cohort though, mean BMI was 27.3 but did not preclude reliable MDCT assessment. Hence, MDCT imaging might be less affected by increased BMI than TTE imaging.

Leaflet motion can be evaluated with MDCT as demonstrated previously [15]. The maximal difference between the measured angles and manufacturer values was $15^{\circ}$ for the $\mathrm{ON}$ $\mathrm{X}$ valve but only $6^{\circ}$ for all other mechanical valves. Although the leaflets of the $\mathrm{ON}-\mathrm{X}$ valve are designed to open to $90^{\circ}$, our study demonstrated a more reduced opening angle for this valve type in all patients, without any signs of dysfunction or elevated transprosthetic gradients. This phenomenon has previously also been found using fluoroscopy in patients with aortic On-X valves [17] and in phantom setting in mitral ON$X$ valves [18]. For biological leaflets, Chenot et al. [5] previously measured leaflet angles in a variety of biological valve types. In our cohort, the leaflets of the biological Perimount
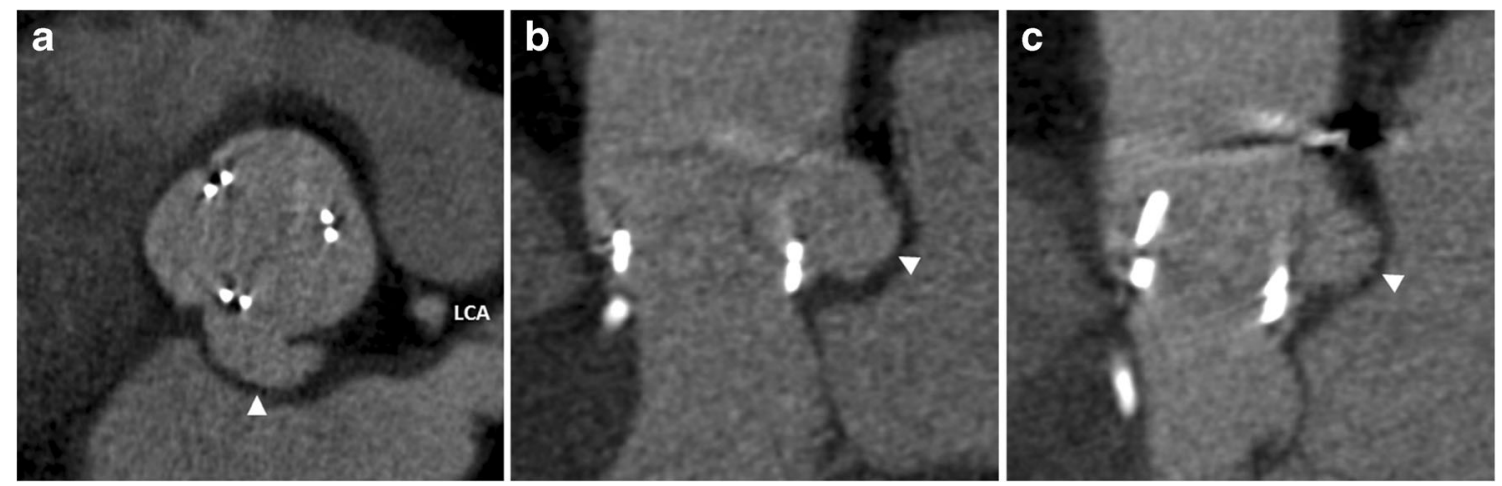

Fig. 4 Detection of a pseudoaneurysm. Multidetector-row computed tomography (MDCT) detected a pseudoaneurysm on the supraprosthetic site near the former left and non-coronary cusp in a 74year-old male patient 6 weeks after aortic Perimount 25-mm implantation. The pseudoaneurysm (arrowheads) of approximately $22 \times$ $9 \times 13 \mathrm{~mm}$ is presented on the in-plane (a) and the perpendicular plane MDCT views $(\mathbf{b}, \mathbf{c})$. No pathology or periprosthetic regurgitation was found with transthoracic echocardiography. $L C A$ left coronary artery 

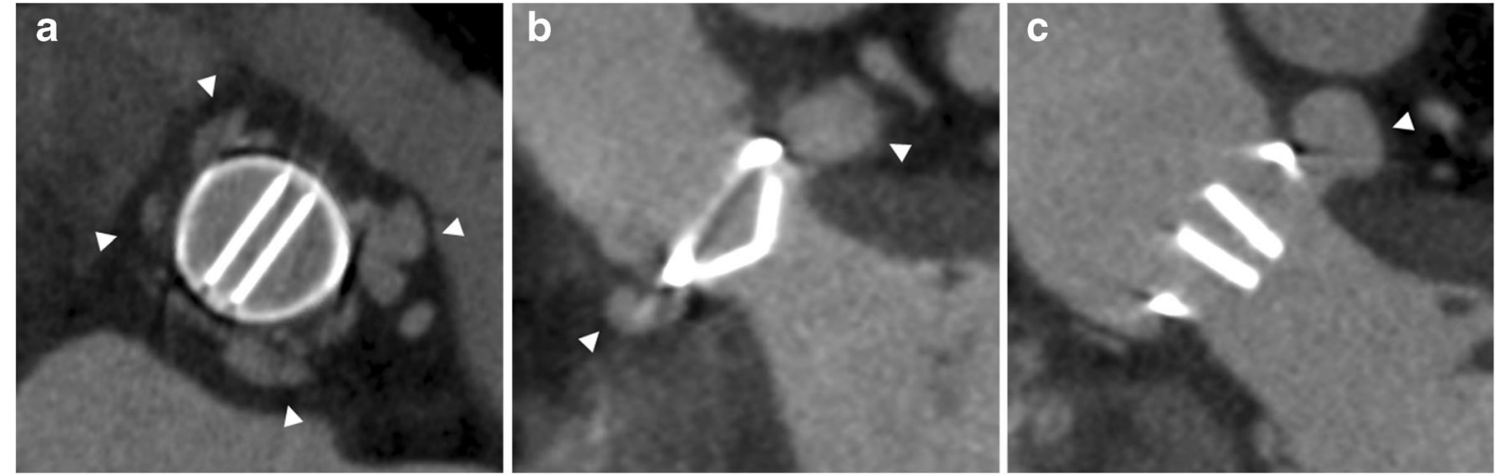

Fig. 5 Unexpected pathology requiring redo valve surgery. Fifty-oneyear old female patient after valve replacement surgery with the mechanical St. Jude 23-mm prosthesis in aortic position. Multidetectorrow computed tomography imaging (a-c) showed extended

valves were not assessable. Whereas during follow-up the biological leaflets may thicken and become more visible, in normal settings early after implantation these leaflets were too thin for MDCT assessment. Nevertheless, MDCT allowed assessment of both aortic and mitral valve leaflet angles while conventional fluoroscopy has shown significant problems in positioning the beam perpendicular to the valve leaflets in certain anatomical valve orientations [17, 19]. Another remarkable MDCT finding was the induration of the periaortic fat tissue in $83 \%$ of normal follow-up patients with aortic valves. In patients suspected of PHV endocarditis, the appearance of this area is of paramount importance for diagnosis. Apparently, 6 weeks post uncomplicated aortic valve surgery, the periaortic fat tissue is still disturbed in the majority of patients and can be considered a normal baseline finding. However, it is not known how long this periaortic induration persists. In case of early postoperative endocarditis suspicion pseudoaneurysm formation (arrowheads) and valve dehiscence eventually requiring redo surgery. Transthoracic echocardiography detected only a trace of periprosthetic regurgitation, stated as clinically not relevant

it might be difficult to distinguish a physiological state from pathological conditions based solely on the fat tissue's MDCT appearance. Further studies are needed to establish the duration of periaortic induration. In future setting, additional $18 \mathrm{~F}$ fluorodeoxyglucose positron emission tomography might possibly aid in the diagnostic work-up of endocarditis patients by showing increased metabolic uptake values [16].

Although TTE remains important for providing haemodynamic information, MDCT demonstrated several findings that were not detected by TTE. For example, MDCT found a tilted position of three aortic PHVs, which has been related to an increased transprosthetic gradient [4]. In patients with suspected PHV dysfunction, MDCT may aid in diagnostic work-up by detecting a tilted position as the cause of obstruction. Furthermore, MDCT detected prominent subprosthetic tissue in one patient and a large pseudoaneurysm and malignant coronary anomaly in another patient. Above all, MDCT

Table 4 Morphological prosthetic valve assessment by multidetector-row computed tomography

\begin{tabular}{|c|c|c|c|c|}
\hline & \multicolumn{2}{|l|}{ Aortic PHV } & \multicolumn{2}{|l|}{ Mitral PHV } \\
\hline & Mechanical $N=25$ & Biological $N=15$ & Mechanical N=8 & Biological $N=1$ \\
\hline Pledgets visible, n (\%) & $17(68 \%)$ & $12(80 \%)$ & $6(75 \%)$ & $1(100 \%)$ \\
\hline Periaortic fat tissue induration & $22(88 \%)$ & $11(73 \%)$ & $\mathrm{n} / \mathrm{a}$ & $\mathrm{n} / \mathrm{a}$ \\
\hline Leaflet thickening & - & - & - & - \\
\hline Thrombus & - & - & - & - \\
\hline Subprosthetic tissue & - & $1(7 \%)$ & - & - \\
\hline Vegetations & - & - & - & - \\
\hline Abscesses & - & - & - & - \\
\hline Pseudoaneurysms & $1(4 \%)$ & $1(7 \%)$ & - & - \\
\hline Valve dehiscence & $1(4 \%)$ & - & - & - \\
\hline Valve angulation* & $1(4 \%)$ & $2(13 \%)$ & - & - \\
\hline Abnormal pericardial effusion & $1(4 \%)$ & $2(13 \%)$ & - & - \\
\hline Pericardial haeamatoma & $2(8 \%)$ & $1(7 \%)$ & - & - \\
\hline
\end{tabular}

$n / a$ not applicable, $P H V$ prosthetic heart valve

* To the left ventricular outflow tract axis 
detected severe valve dehiscence requiring redo surgery while TTE only showed a trace of insignificant periprosthetic regurgitation. Thus, MDCT showed incremental value over echocardiography in the assessment of anatomical structures near the prosthesis and in the detection of related pathology such as pseudoaneurysms. Recently, we demonstrated the feasibility of MDCT for non-invasive coronary artery and bypass graft assessment in PHV dysfunction patients [20].

The incidental (pathological) findings in this study may need to be confirmed in larger series in order to assess their long-term significance and to clarify the role of MDCT as a screening modality. Because of the limitations of radiation exposure, screening with MDCT may only be possible with a certain amount of clinical suspicion. On the other hand, the utilization of dose reduction strategies such as iterative image reconstruction algorithms allows a far more widespread use of MDCT. We did not incorporate these techniques in the current study. Prospectively ECG-triggered MDCT acquisitions may also save radiation exposure, but they do not provide dynamic information on leaflet excursions. As shown in this study, the optimal systolic and diastolic phases for PHV assessment differ substantially among patients. Hence, selecting the best phase in advance as required with prospectively triggered imaging can be very challenging.

A strength of this study is the prospective cohort. No selection was made based on clinical signs. Thus, a random sample of normal patients shortly after successful valve replacement surgery is presented. All patients underwent TTE and MDCT imaging in the same hospital centre using the same devices and software for all patients. Furthermore, MDCT and TTE were performed on the same day in $98 \%$ of patients, thereby virtually excluding differences in results due to time effect and/or left ventricular filling conditions.

A limitation of the current study is that we could not assess all currently available prosthetic valves. However, based on available in vitro and in vivo MDCT studies we do not expect significant differences with other contemporary valves [14]. Furthermore, patients with an impaired renal function were excluded to reduce the risk of contrast-induced nephropathy. For logistic purposes, the renal function was assessed early after cardiothoracic surgery (before discharge). It is probable that at 6 weeks after implantation renal function has recovered and contrast-enhanced imaging can be performed in more patients. No beta blockers were administered before MDCT acquisition, which may have affected image quality.

\section{Conclusion}

Six weeks after valve replacement surgery, MDCT allowed evaluation of relevant anatomical regions in all PHVs in both aortic and mitral position despite mild to moderate artefacts, and presented baseline findings such as tilted aortic PHV positions and prominent pericardial haematoma. A major additional finding was induration of the periaortic fat tissue present in $83 \%$ of patients after uncomplicated aortic valve replacement. The use of MDCT as a complementary imaging tool for follow-up in postoperative patients is feasible, and revealed three cases of clinically relevant pathology that were not detected by TTE imaging. Further studies are needed to assess whether early abnormalities with MDCT in seemingly normally functioning valves are as frequent as in this series.

Acknowledgments The authors thank Jakub J. Regieli for his help regarding echocardiography analysis. The scientific guarantor of this publication is Dr. R.P.J. Budde. The authors of this manuscript declare relationships with the following companies: Lex. A. van Herwerden is a clinical research consultant for St. Jude Medical Inc., St. Paul, MN, USA. This study has received funding through a grant from the Dutch Heart Foundation, The Netherlands [Grant number 2009B014]. No complex statistical methods were necessary for this paper. Institutional Review Board approval was obtained. Written informed consent was obtained from all subjects (patients) in this study. One study subject has been previously reported in: Tanis W, Scholtens A, Habets J et al (2013) CT angiography and (1)(8)F-FDG-PET fusion imaging for prosthetic heart valve endocarditis. JACC Cardiovasc Imaging 6:1008-1013

One patient has previously been reported in an imaging vignette, concerning a FDG-PET/CT imaging series for prosthetic valve endocarditis, whereas in the current study this patient was imaged with CTA for baseline postsurgical imaging characteristic purposes.

Methodology: prospective, diagnostic study, performed at one institution.

Open Access This article is distributed under the terms of the Creative Commons Attribution-NonCommercial 4.0 International License (http:// creativecommons.org/licenses/by-nc/4.0/), which permits any noncommercial use, distribution, and reproduction in any medium, provided you give appropriate credit to the original author(s) and the source, provide a link to the Creative Commons license, and indicate if changes were made.

\section{References}

1. Vahanian A, Alfieri O, Andreotti F et al (2012) Guidelines on the management of valvular heart disease (version 2012): the Joint Task Force on the Management of Valvular Heart Disease of the European Society of Cardiology (ESC) and the European Association for Cardio-Thoracic Surgery (EACTS). Eur J Cardiothorac Surg 42:S1-S44

2. Nishimura RA, Otto CM, Bonow RO et al (2014) 2014 AHA/ACC guideline for the management of patients with valvular heart disease: executive summary: a report of the American College of Cardiology/American Heart Association Task Force on Practice Guidelines. J Am Coll Cardiol 63:2438-2488

3. Habets J, Tanis W, van Herwerden LA et al (2014) Cardiac computed tomography angiography results in diagnostic and therapeutic change in prosthetic heart valve endocarditis. Int $\mathrm{J}$ Cardiovasc Imaging 30:377-387

4. Symersky P, Budde RP, de Mol BA, Prokop M (2009) Comparison of multidetector-row computed tomography to echocardiography and fluoroscopy for evaluation of patients with mechanical prosthetic valve obstruction. Am J Cardiol 104:1128-1134

5. Chenot F, Montant P, Goffinet $\mathrm{C}$ et al (2010) Evaluation of anatomic valve opening and leaflet morphology in aortic valve 
bioprosthesis by using multidetector CT: comparison with transthoracic echocardiography. Radiology 255:377-385

6. Fagman E, Perrotta S, Bech-Hanssen O et al (2012) ECG-gated computed tomography: a new role for patients with suspected aortic prosthetic valve endocarditis. Eur Radiol 22:2407-2414

7. Teshima H, Hayashida N, Fukunaga S et al (2004) Usefulness of a multidetector-row computed tomography scanner for detecting pannus formation. Ann Thorac Surg 77:523-526

8. Habets J, Tanis W, Reitsma JB et al (2015) Are novel non-invasive imaging techniques needed in patients with suspected prosthetic heart valve endocarditis? A systematic review and meta-analysis. Eur Radiol. doi:10.1007/s00330-015-3605-7

9. Zoghbi WA, Chambers JB, Dumesnil JG et al (2009) Recommendations for evaluation of prosthetic valves with echocardiography and doppler ultrasound: a report From the American Society of Echocardiography's Guidelines and Standards Committee and the Task Force on Prosthetic Valves, developed in conjunction with the American College of Cardiology Cardiovascular Imaging Committee, Cardiac Imaging Committee of the American Heart Association, the European Association of Echocardiography, a registered branch of the European Society of Cardiology, the Japanese Society of Echocardiography and the Canadian Society of Echocardiography, endorsed by the American College of Cardiology Foundation, American Heart Association, European Association of Echocardiography, a registered branch of the European Society of Cardiology, the Japanese Society of Echocardiography, and Canadian Society of Echocardiography. J Am Soc Echocardiogr 22:975-1014

10. Otto CM, Pearlman AS, Comess KA, Reamer RP, Janko CL, Huntsman LL (1986) Determination of the stenotic aortic valve area in adults using Doppler echocardiography. J Am Coll Cardiol 7:509-517
11. Zoghbi WA, Farmer KL, Soto JG, Nelson JG, Quinones MA (1986) Accurate noninvasive quantification of stenotic aortic valve area by Doppler echocardiography. Circulation 73:452-459

12. Deak PD, Smal Y, Kalender WA (2010) Multisection CT protocols: sex- and age-specific conversion factors used to determine effective dose from dose-length product. Radiology 257:158-166

13. de Heer LM, Habets J, Kluin J et al (2013) Assessment of a transcatheter heart valve prosthesis with multidetector computed tomography: in vitro and in vivo imaging characteristics. Int J Cardiovasc Imaging 29:659-668

14. Habets J, Symersky P, van Herwerden LA et al (2011) Prosthetic heart valve assessment with multidetector-row CT: imaging characteristics of 91 valves in 83 patients. Eur Radiol 21:1390-1396

15. Suchá D, Symersky P, Vonken EJ, Provoost E, Chamuleau SA, Budde RP (2014) Multidetector-row computed tomography allows accurate measurement of mechanical prosthetic heart valve leaflet closing angles compared with fluoroscopy. J Comput Assist Tomogr 38:451-456

16. Tanis W, Scholtens A, Habets J et al (2013) CT angiography and (1)(8)F-FDG-PET fusion imaging for prosthetic heart valve endocarditis. JACC Cardiovasc Imaging 6:1008-1013

17. Cianciulli TE, Lax JA, Beck MA et al (2005) Cinefluoroscopic assessment of mechanical disc prostheses: its value as a complementary method to echocardiography. J Heart Valve Dis 14:664-673

18. Feng Z, Nakamura T, Fujimoto T, Umezu M (2002) In vitro investigation of opening behavior and hydrodynamics of bileaflet valves in the mitral position. Artif Organs 26:32-39

19. Montorsi P, Arena V, Muratori M et al (1996) Fluoroscopic functional evaluation of bileaflet prostheses: effect of different intraoperative valve orientation. Am J Card Imaging 10:101-107

20. Tanis W, Suchá D, Laufer W et al (2015) Multidetector-row computed tomography for prosthetic heart valve dysfunction: is concomitant non-invasive coronary angiography possible before redo-surgery? Eur Radiol 25:1623-1630 